\title{
Direct navigated 3D ultrasound for resection of brain tumors: a useful tool for intraoperative image guidance
}

\author{
Aliasgar V. Moiyadi, MCh, and Prakash Shetty, MCh \\ Division of Neurosurgery, Department of Surgical Oncology, Tata Memorial Centre, Mumbai, India
}

OBJECTIVE Navigated 3D ultrasound is a novel intraoperative imaging adjunct permitting quick real-time updates to facilitate tumor resection. Image quality continues to improve and is currently sufficient to allow use of navigated ultrasound (NUS) as a stand-alone modality for intraoperative guidance without the need for preoperative MRI.

METHODS The authors retrospectively analyzed cases involving operations performed at their institution in which a 3D ultrasound navigation system was used for control of resection of brain tumors in a "direct" 3D ultrasound mode, without preoperative MRI guidance. The usefulness of the ultrasound and its correlation with postoperative imaging were evaluated.

RESULTS Ultrasound was used for resection control in 81 cases. In 53 of these 81 cases, at least 1 intermediate scan (range 1-3 intermediate scans) was obtained during the course of the resection, and in 50 of these 53 cases, the result prompted further resection. In the remaining 28 cases, intermediate scans were not performed either because the first ultrasound scan performed after resection was interpreted as showing no residual tumor $(n=18)$ and resection was terminated or because the surgeon intentionally terminated the resection prematurely due to the infiltrative nature of the tumor and extension of disease into eloquent areas $(n=10)$ and the final ultrasound scan was interpreted as showing residual disease. In an additional 20 cases, ultrasound navigation was used primarily for localization and not for resection control, making the total number of NUS cases where radical resection was planned 101. Gross-total resection (GTR) was planned in 68 of these 101 cases and cytoreduction in 33. Ultrasound-defined GTR was achieved in 51 (75\%) of the cases in which GTR was planned. In the remaining 17, further resection had to be terminated (despite evidence of residual tumor on ultrasound) because of diffuse infiltration or proximity to eloquent areas. Of the 33 cases planned for cytoreduction, NUS guidance facilitated ultrasound-defined GTR in 4 cases. Overall, ultrasound-defined GTR was achieved in $50 \%$ of cases (55 of 111). Based on the postoperative imaging (MRI in most cases), GTR was achieved in 58 cases (53\%). Final (postresection) ultrasonography was documented in 78 cases. The findings were compared with the postoperative imaging to ascertain concordance in detecting residual tumor. Overall concordance was seen in 64 cases $(82.5 \%)$, positive concordance was seen in $33(42.5 \%)$, and negative in $31(40 \%)$. Discordance was seen in 14 caseswith ultrasound yielding false-positive results in 7 cases and false-negative results in 7 cases. Postoperative neurological worsening occurred in 15 cases (13.5\%), and in most of these cases, it was reversible by the time of discharge.

CONCLUSIONS The results of this study demonstrate that 3D ultrasound can be effectively used as a stand-alone navigation modality during the resection of brain tumors. The ability to provide repeated, high-quality intraoperative updates is useful for guiding resection. Attention to image acquisition technique and experience can significantly increase the quality of images, thereby improving the overall utility of this modality.

http://thejns.org/doi/abs/10.3171/2015.12.FOCUS15529

KEY WORDS navigated ultrasound; stand alone; intraoperative imaging; glioma surgery; navigation

$\mathrm{R}$ ADICAL resection is essential for improving outcomes in glioma surgery. ${ }^{21,27}$ Mere reliance on intraoperative microsurgical techniques is probably not sufficient for consistently achieving this goal, as the visualized tumor borders are often indistinct, even when viewed through a surgical microscope. Navigation sys- tems provide a roadmap for surgery by integrating preoperative images (usually MRI) and registering them to the patient..$^{36}$ The use of these systems during tumor resection can be divided simply into 2 phases - the first phase includes lesion localization (this includes planning of the bony exposure as well as localization of the tumor mass 
for selecting the trajectory of entry), and the second phase is for actual control during the resective phase of the surgery, when repeated updates are desirable. The limitation of navigation systems is primarily related to their inability to follow the dynamic changes in intraoperative anatomy as resection proceeds. ${ }^{18}$ This has led to various strategies for intraoperative imaging, such as MRI, CT, and ultrasonography, to update tumor-related information in real time. ${ }^{2}$ Although intraoperative MRI would be ideal, it is not widely available and is often prohibitively expensive. Intraoperative ultrasonography, on the other hand, is readily available..$^{14}$ Advances in ultrasound technology have resulted in navigation solutions that overcome many of the limitations of conventional 2D ultrasound. Navigated ultrasound (NUS) is a novel technique for intraoperative imaging using tracked ultrasound images (both 2D and 3D) for guidance and improving orientation. Many reports describe ultrasound as being used along with preoperative MRI for the purpose of navigation, essentially using image coregistration to continue utilizing preoperative MRI information (either by visual estimation of brain shift or, less commonly, by automatic brain-shift correction).$^{8,9,13,22,24}$ Navigated $3 \mathrm{D}$ ultrasound is a type of NUS using 3D ultrasound volumes. ${ }^{33}$ It can be used in conjunction with preoperative MR images or as a stand-alone modality. There are advantages to using NUS directly. We describe our experience with using direct 3D NUS without preoperative MRI guidance during tumor resection.

\section{Methods}

This was a retrospective analysis. We have been using the SonoWand system (SonoWand AS) for navigated 3D ultrasound since June 2011. This system has been previously described in detail.,16 Although it can function as a routine navigation platform using preoperative MR images, it includes the capability of acquiring reformatted 3D ultrasound images that can be tracked. The 3D ultrasound can be used in conjunction with preoperative MR images, or it can be used by itself as a stand-alone direct navigated ultrasound (dNUS) option. For this study, we queried a database that includes all cases of surgically treated brain tumors for which 3D ultrasound navigation was used and selected for analyses all studies in which the dNUS mode of function was used. The patients' clinical characteristics, ultrasound parameters, extent of resection, and postoperative outcomes were recorded. Descriptive data were reported as percentages. The probes used included the 8FPA (3- to 8-MHz phased array, $25 \times 17-\mathrm{mm}$ footprint), 10FPA-MC (5- to $10-\mathrm{MHz}$ phased array, $15 \times$ 13-mm footprint), and 12FLA (6- to 12-MHz linear array, $32 \times 11-\mathrm{mm}$ footprint). The evaluation of the ultrasound features included the type of probe used, the resolution of the images, the number of scans (initial and intermediate, as well as final) performed, and the decision of the surgeon at each time point. This information was extracted from the operative records, where this is specifically documented as a routine protocol. The final ultrasound scan interpretation was considered in identifying ultrasounddefined gross-total resections (GTRs). Postoperative MRI studies were obtained in the majority of cases. Whenever
MRI was not possible, CT scans were obtained. Postoperative imaging was semiquantitatively recorded as GTR (no residual tumor), subtotal resection $(<50 \%$ residual tumor), or partial resection ( $>50 \%$ residual tumor). Ultrasound and MRI (or CT) findings were correlated to find the concordance.

\section{Results}

A total of 202 operations were performed using the 3D ultrasound system between June 2011 and May 2015 at our institution. Of these, 111 cases were identified where only dNUS was used. These 111 cases are the subject of further analysis in this paper. The patients' sex, tumor characteristics, and ultrasound features for these cases are presented in Table 1. Most of the tumors were high-grade gliomas. Routine microneurosurgical principles were used for all cases. Functional monitoring (with awake craniotomy)

TABLE 1. Table depicting the clinicoradiological features of the patient cohort $(n=111)$

\begin{tabular}{|c|c|}
\hline Characteristic & No. of Patients (\%) \\
\hline \multicolumn{2}{|l|}{ Sex } \\
\hline Male & $75(68)$ \\
\hline Female & $36(32)$ \\
\hline \multicolumn{2}{|l|}{ Intended resection } \\
\hline Gross total & $68(61)$ \\
\hline Cytoreduction & $33(30)$ \\
\hline Biospy & $10(9)$ \\
\hline \multicolumn{2}{|c|}{ Tumor delineation (on preop MRI) } \\
\hline Good & $42(38)$ \\
\hline Moderate & $55(50)$ \\
\hline Poor & $14(12)$ \\
\hline \multicolumn{2}{|l|}{ Eloquence } \\
\hline Noneloquent & $31(28)$ \\
\hline Close (up to $5 \mathrm{~mm}$ ) & $51(46)$ \\
\hline Eloquent & $29(26)$ \\
\hline \multicolumn{2}{|l|}{ US image quality } \\
\hline Good & $54(49)$ \\
\hline Fair & $47(43)$ \\
\hline Poor & $10(8)$ \\
\hline \multicolumn{2}{|l|}{ Histology } \\
\hline High-grade glioma & $75(68)$ \\
\hline Low-grade glioma & $12(11)$ \\
\hline Metastasis & $3(2.6)$ \\
\hline Meningioma & $8(7)$ \\
\hline PNET/medulloblastoma & $3(2.6)$ \\
\hline Lymphoma & $3(2.6)$ \\
\hline Other & $7(6)$ \\
\hline \multicolumn{2}{|l|}{ Extent of resection } \\
\hline Gross total & $58(53)$ \\
\hline Near total & $17(15)$ \\
\hline Subtotal & $23(21)$ \\
\hline Partial & $13(11)$ \\
\hline
\end{tabular}

PNET = primitive neuroectodermal tumor; US = ultrasound. 
was performed when needed. As part of a multimodal strategy, 5-aminolevulinic acid-based fluorescence guidance was used in 16 of these cases. For all cases, the NUS was used for decision making in conjunction with the surgeon's intraoperative judgment and intraoperative functional monitoring findings. The ultrasound probe 8FPA was used in the 94 of the 111 cases. Linear (12FLA) and minicraniotomy (10FPA-MC) probes were used less often. The power Doppler function of the SonoWand system was used to identify important vessels in the vicinity of the tumors.

NUS was used for resection control in 81 of the 111 cases. Of the remaining 30 cases, 10 involved procedures that were intended to be only biopsies (NUS was used for frameless ultrasound-guided biopsy). All 10 yielded a positive diagnosis (confirmed at intraoperative frozen section). In the 20 other cases, resection was planned, but NUS was not used after the initial ultrasound acquisition either because it was not necessary $(n=16,12$ metastases or extra-axial lesions, 4 high-grade gliomas where fluorescence-guided resections were performed) or was technically inadequate ( $\mathrm{n}=4$, all gliomas). The technical failures resulted from inadequate insonation due to positioning problems during the surgery. In all 30 of these cases, NUS was used only for lesion localization. In the 81 cases for which NUS was used for resection control, at least 1 intermediate scan (range 1-3) was obtained during the course of the resection in 53 cases, prompting further resection in 50. In this subgroup, 10 cases involved highgrade gliomas for which intraoperative fluorescence was also used. In the remaining 28 cases, intermediate scans were not performed either because the first ultrasound scan obtained after resection was interpreted as showing no residual tumor $(n=18$, in 2 cases fluorescence was also used as a guide for resection) and resection was terminated or because the surgeon intentionally terminated the resection prematurely due to the infiltrative nature and extension of disease into eloquent areas $(n=10)$ and the final ultrasound was interpreted as showing residual disease. Figures 1-3 show typical representative cases where dNUS was used. Figure 4 shows the outline of the results.

Of the 111 cases where dNUS was used, debulking was intended in 101 (GTR planned in 68 and cytoreduction in 33; Table 1). In the intended GTR group, ultrasounddefined GTR was achieved in 51 cases $(75 \%)$. In the remaining 17 cases, further resection had to be abandoned (despite ultrasound evidence of residual tumor) because of diffuse infiltration or proximity to eloquent areas. Of the 33 cases in which cytoreduction was planned, NUS guidance facilitated ultrasound-defined GTR in 4 (12\%). Overall, ultrasound-defined GTR was achieved in 55 (50\%) of 111 cases. Based on the postoperative imaging (MRI in most cases), GTR was achieved in 58 cases (53\%) (Table 1). Final (postresection) ultrasound findings were documented in 78 cases. These results were compared with the results of postoperative imaging to ascertain concordance in detecting residual tumor. Overall concordance was seen in 64 cases $(82.5 \%$ overall; positive concordance in 33 [42.5\%] and negative in 31 [40\%]). Discordance was seen in 14 cases: 7 in which ultrasound produced false-positive results and 7 in which it produced false-negative results.
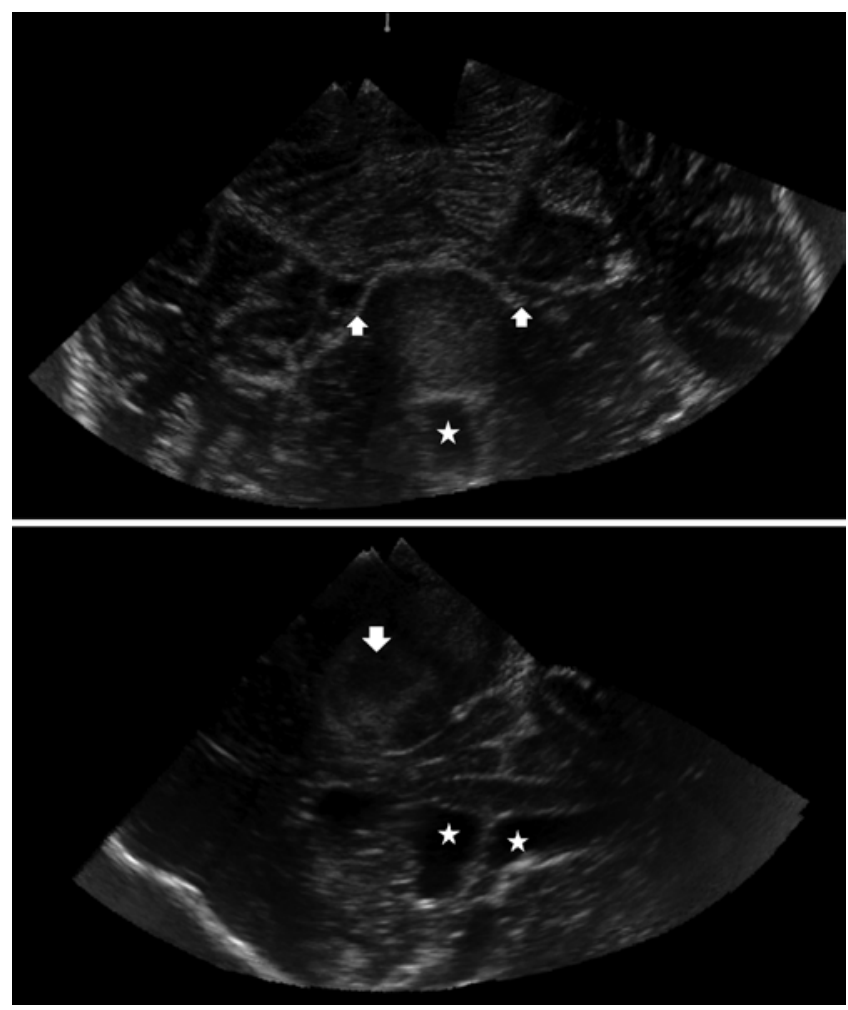

FIG. 1. Representative high-resolution intraoperative ultrasound images. Upper: A tectal plate glioma approached through the supracerebellar route. The well-circumscribed uniformly hyperechoic tumor is seen in the center of the image rimmed by the hyperechoic tentorial hiatus (arrows). The pineal recess of the dilated posterior third ventricle is seen beyond the tumor (star), and the characteristic pattern of the cerebellar folia is clearly appreciated in front of the tumor. Lower: A right frontal glioma approached through a right frontal craniotomy. Beyond the tumor (arrow), the bilateral frontal horns (stars) and falx (central vertical hyperechoic line) are clearly seen. In both images, a wide, large-volume insonation allowed appreciation of normal anatomy, facilitating orientation, even in the absence of MR-based navigation.

Postoperative worsening of neurological condition occurred in 15 cases $(13.5 \%)$. In 13 of these cases, the worsening was transient and the patients recovered by the time of discharge; in 2 cases, it was prolonged and persisted at the 1-month follow-up.

\section{Discussion}

Conventional navigation systems are essentially based on preoperatively acquired CT or MR images. They facilitate the accurate planning and execution of tailored craniotomies. They are also very useful for localizing the lesion and planning the optimal entry path to the lesion. However, one must remember that all navigation systems require image-to-patient registration, which introduces the element of "registration error." Sometimes technical failures or mishaps (such as movement of the reference frame) may result in inadvertent complete failure of the navigation, literally leaving the surgeon stranded without a backup alternative. Moreover, once the dura is opened (and occasionally, even before that) "brain shift" sets in, rendering the preoperatively acquired images inaccurate and therefore no longer reliable for further navigation. ${ }^{18,19}$ 

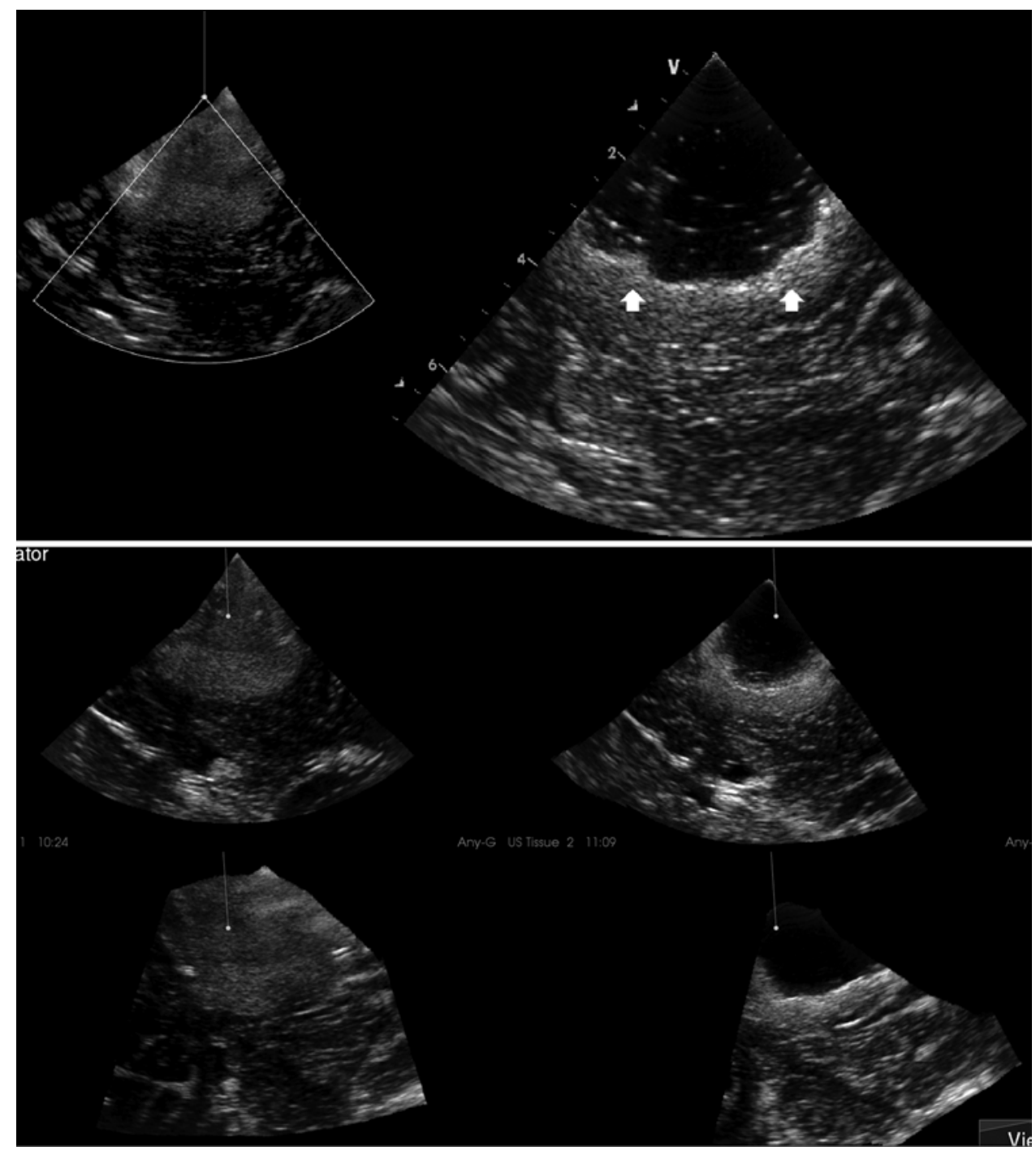

FIG. 2. Upper: Screenshot of the intraoperative ultrasound scan obtained after partial tumor debulking. Residual tumor is seen as a hyperechoic rim (white arrows). The left side of the screenshot shows the plane of acquisition of this image with respect to the preresection US (white sector outline superimposed on the preresection tumor image). Note that the sector outline is not exactly aligned to the preoperative image, implying that the plane of visualization of the real-time image displayed on the right does not correspond to the preresection scan plane on the left. Therefore, any estimation of location of residual tumor will not be accurate if only 2D images are used. Lower: The same images as above now acquired with navigated 3D ultrasound and co-displayed in 2 orthogonal identically corresponding planes, allowing for point-to-point correlation of image characteristics.

Thus, the use of routine navigation is questionable if its applicability beyond the stage of craniotomy is limited, especially considering that appropriate craniotomies are possible to plan with a good knowledge of surface anatomy after carefully studying the preoperative images. The use of innovative and improvised techniques can also help in planning accurate craniotomies without the need for navigation..$^{1,30}$ The additional time (and expense) of performing a navigated craniotomy may not be justifiable in many high-volume centers, particularly in resource-constrained settings. Whereas navigation is not indispensable for planning the craniotomy, it is crucial during the subsequent resective phase of the surgery for intra-axial tumors. Hence, some form of intraoperative imaging is preferable. ${ }^{2}$ Intraoperative ultrasonography provides reliable real-time updates of the operative field ("live anatomy").4,10,14,25,34,35,37
The limitation of ultrasound in delineating residual tumor is mainly related to 2 issues: image quality and anatomical orientation. Recent advances in ultrasound technology have significantly improved resolution. Its optimal use, however, requires some training and experience, which can be easily acquired. Furthermore, it is known that the image resolution and quality of the ultrasound images decrease as resection proceeds. ${ }^{26}$ This is primarily because of artifacts introduced by the surgical manipulation. Many strategies have been described to reduce these artifacts, making it possible to eliminate the majority. ${ }^{28}$ The bigger problem is probably that of lack of anatomical orientation. Traditionally, B-mode ultrasound images are displayed in planar format, with the view limited to the field insonated (which may be a sector or a rectangular display depending on the probe used) and in the plane of insonation (which 

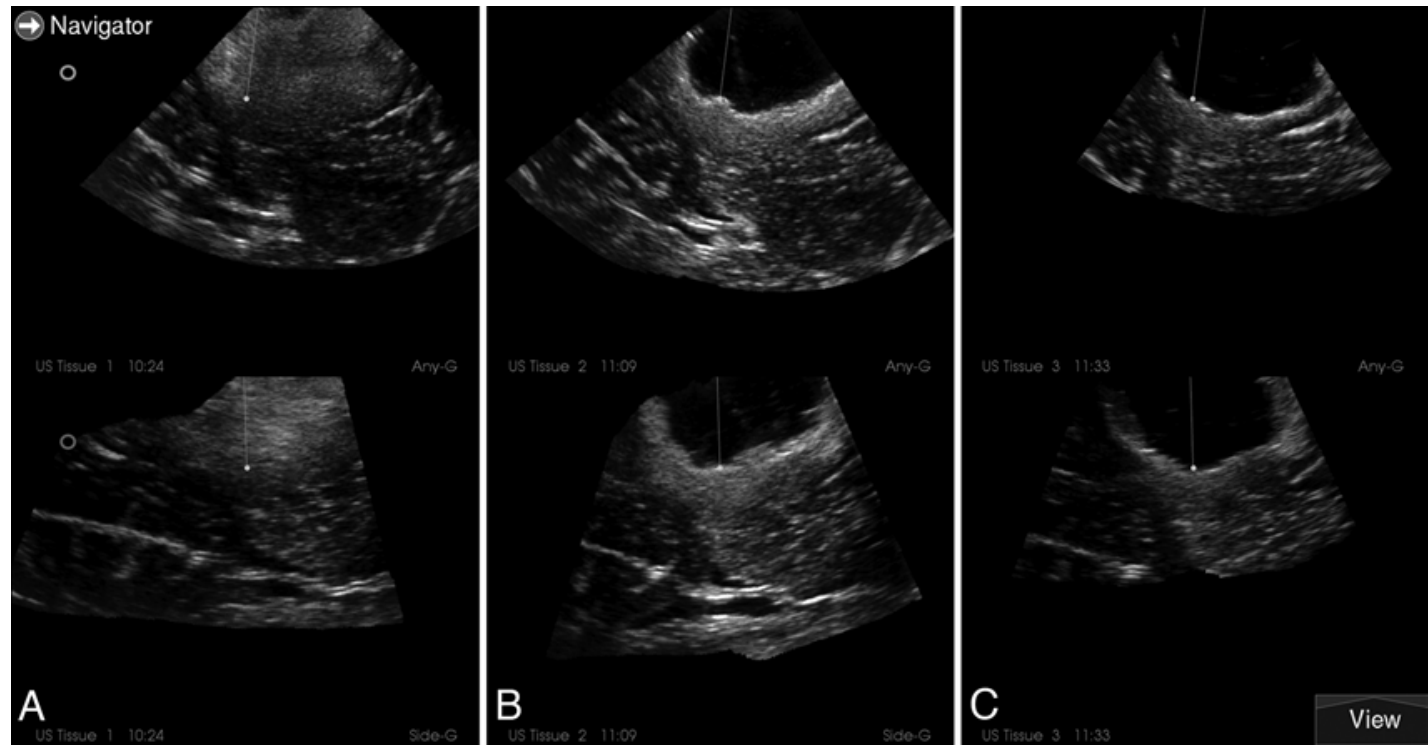

FIG. 3. 3D ultrasound images (from the same case as the images in Fig. 2) obtained at different stages of the surgery (A, preresection; B, during resection; C, postresection) and co-displayed in identical planes. This allows pinpoint localization of exact site of (suspected) residual tumor and its localization using a navigator tool (straight white line in each images indicates the navigator position).

often is oblique and not the traditional axial, coronal, and sagittal views most neurosurgeons are accustomed to). $\mathrm{Ab}$ sence of known anatomical landmarks within this plane would make it very difficult to understand the orientation. Even if the orientation can be deciphered, one needs a photographic memory and a very accurate sense of position to transfer the ultrasound image information into the operative space, especially when looking for small areas of residual tumor (Figs. 2 and 3). Technically, performing the ultrasound scan in true real time (simultaneously as the surgery is proceeding) can overcome this problem. However, it is logistically challenging (bigger exposure for simultaneous ultrasound and surgical instrument placement) and may often be inaccurate if the tip of the surgical tool is not exactly in the plane of the insonation..$^{32}$ Navigated ultrasound is essentially a "tracked" ultrasound. Just as preoperative MR images can be navigated, integrating intraoperative ultrasound into navigation can help track and navigate the ultrasound images. Both customized integration of ultrasound into commercially available navigation systems $^{5,8,9,22}$ and precalibrated ultrasound navigation ${ }^{7}$ solutions are available. The navigated ultrasound may be 2D (planar) or 3D (volumetric). Navigated 2D ultrasound is usually used in conjunction with preoperative MR images. 3D ultrasound can be acquired either by using specialized (and motorized) 3D probes ${ }^{17}$ or by reconstructing a 3D ultrasound volume from multiple stacked 2D ultrasound images acquired using a tracked ultrasound probe.,11,29 Whereas tracking is essential for reconstructing 3D volumes from multiple 2D images, it is not required for 3D probes that directly acquire 3D volumes. However, if the resultant volume is to be practically useful for navigating during surgery, it is essential to be able to track the images. Without the navigable component, even 3D ultrasound is of limited use. ${ }^{3}$ When the ultrasound probe is tracked, the exact spatial coordinates of the ultrasound volume scanned are known, and the system is able to navigate instruments within this volume. Our system uses digitally transferred and reconstructed tracked 3D ultrasound images (SonoWand).

Navigated direct 3D ultrasound eliminates the need for the preoperative MRI altogether. Good-quality ultrasound images have been shown to be comparable to MR images in delineating brain tumors. ${ }^{6,34}$ Therefore, in our opinion, little advantage is gained by using the preoperatively acquired MR images (especially once brain shift has occurred). Also, in many cases, logistical challenges make it difficult to organize preoperative MRI. This is often the case for emergency surgeries or when getting an early preoperative MRI appointment may be difficult (as happens frequently in our center, which has a very busy service, with MRI services being oversubscribed). By acquiring large volumes of insonation (by a combination of adequate exposure, appropriate probe configuration, and optimal scanning technique) to encompass surrounding uninvolved brain regions, ultrasound can provide a panoramic overview of the surgical anatomy, facilitating easy orientation of the surgical field (Fig. 1). 3D ultrasound also provides very good real-time intraoperative angiogram acquisition. Although navigated 2D ultrasound also improves orientation, it is less helpful. The main reason is that, in navigated 2D ultrasound, each time a new image is obtained, it is displayed in relation to the corresponding image plane in MR. Although this helps orientation of the ultrasound slice plane, when repeated serial 2D ultrasound slices are obtained to monitor the progress of a resection, it is impossible to get the same plane of insonation again and again. By acquiring a stack of 2D ultrasound images and reconstructing them into a tracked 3D ultrasound volume, this problem is overcome (Figs. 2 and 3). Once acquired, the 3D volume can be sliced in any desired plane for display (and this can be done repetitively with accu- 


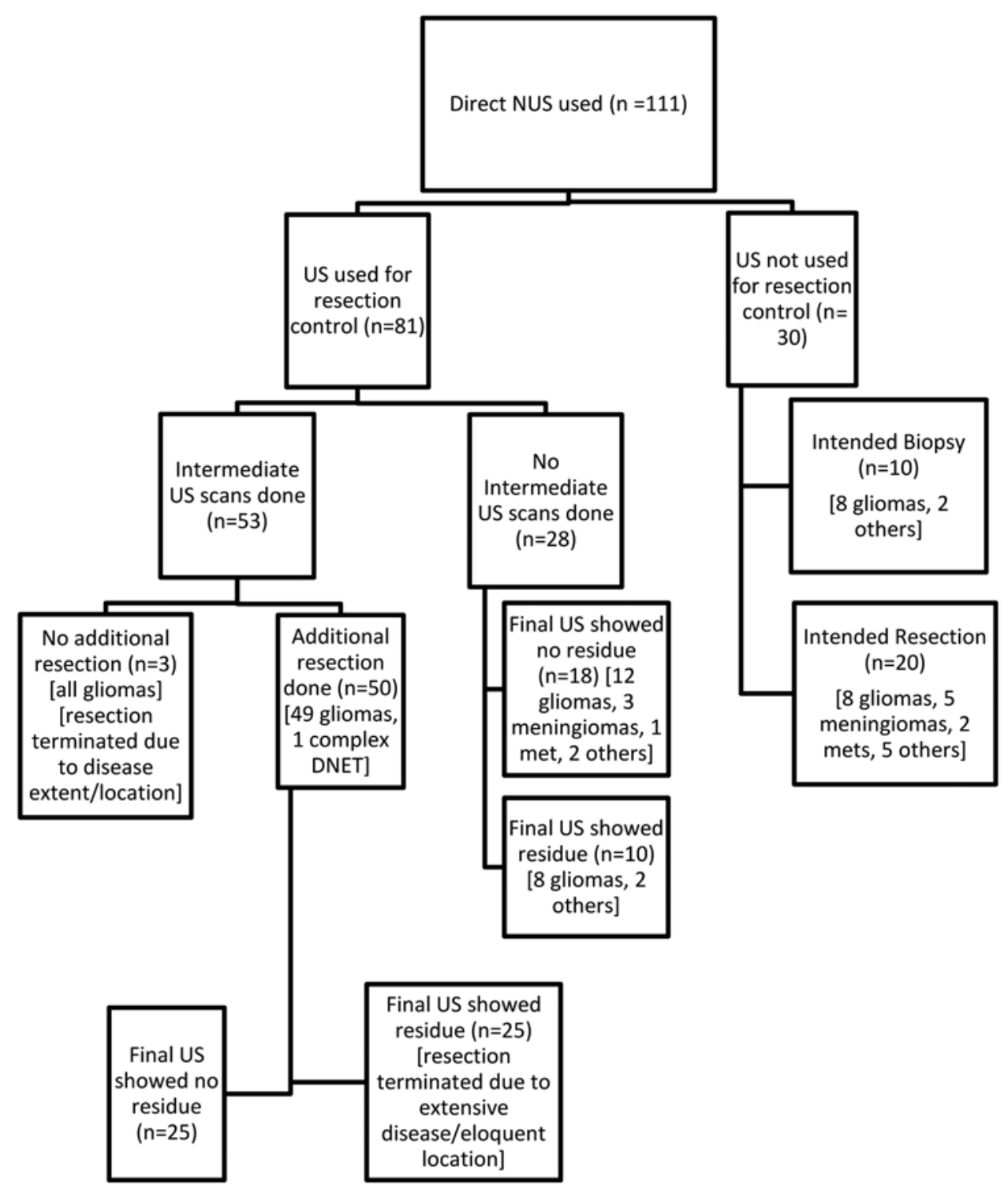

FIG. 4. Flowchart depicting the overall study results. DNET = dysembryoplastic neuroepithelial tumor; met = metastasis; US = ultrasound.

racy), and multiple 3D sets can be compared in the chosen plane. Further, if brain-shift correction algorithms are to be used, 3D ultrasound is essential. ${ }^{23}$

Using this dNUS technique, we were able to achieve intraoperative radical resections in $50 \%$ of cases $(75 \%$ in the intended GTR group). The intraoperative ultrasound findings correlated with postoperative MRI findings in $82.5 \%$ of cases. In the cases in which only biopsy was planned, using dNUS resulted in a diagnostic yield of $100 \%$. Use of direct 3D ultrasound has been previously reported in a few cases. Miller et al. ${ }^{12}$ reported 2 cases of deep-seated lesions in which unexpected failure of the conventional navigation system occurred. The authors resorted to using dNUS for lesion localization and resection control and reported that it was effective. Peredo-Harvey et al. ${ }^{20}$ reported 18 cases (15 of which were tumor resections) in which conventional MRI-based navigation failed (7 cases in which MR images could not be loaded into the navigation system, 5 in which the emergency nature of the procedure did not permit time for preoperative MRI, and 6 other technical failures). These 18 cases constituted $16 \%$ of their navigation cases in the same time period, which effectively means that the failure rate of conventional navigation was $16 \%$ in their series. They went ahead with using the navigated 3D ultrasound (SonoWand) and successfully completed the procedures. The ultrasound was adequate in all cases, and radical (> 99\%) resections were achieved in 11 of 15 cases. In the 4 other tumor resections, the lesions were subtotally excised (95\%-99\% resection) because of proximity to eloquent structures. In 2 of the 18 cases, the system was used for obtaining frameless biopsies, and both biopsies yielded positive diagnoses. Both these reports describe the use of 3D ultrasound as a fallback option after failure of standard navigation. In contrast, the present study describes navigated 3D ultrasound as the preferred firstline modality for intraoperative guidance. We used it for 
both frameless biopsy and resection control. Although it is feasible to perform frameless biopsy using preoperative MRI, we have found it equally convenient to do it under ultrasound guidance through a bur hole with a special minicraniotomy probe (MC probe, SonoWand) and using an intuitive "target" function of the system. The placement of the bur hole can be planned by studying the preoperative scans. All 10 of our cases yielded a positive diagnosis. The major use of dNUS was resection control (80\% of cases), and it is in these cases where its maximum benefit is evident. Solheim et al. ${ }^{31}$ published on a large experience using a similar NUS platform in cases of high-grade gliomas. They used the NUS in 143 of 156 glioma cases in their series. However, in 119 cases, they also used preoperative MR images in addition to the ultrasound, effectively using stand-alone NUS as a single modality in only 24 patients (approximately 16\% of their cases). We have been less reliant on MRI, and hence, almost $50 \%$ of our operations are performed with dNUS. In our experience, dNUS has been extremely effective during brain tumor surgeries.

\section{Limitations and Benefits of dNUS}

We had technical failures in 4 cases (4\%). All of these were because of inadequate insonation due to suboptimal patient position during the surgery, precluding effective filling of the resection cavity with saline for ensuring good acoustic coupling. This may be particularly problematic in awake surgeries, where optimal patient positioning may be difficult to achieve. Ultrasound enabled us to correctly predict residual tumor in $82.5 \%$ of cases. There were still false negatives and false positives, but in our earlier experience, ${ }^{15}$ we have observed that these can be reduced by careful attention to image acquisition, something that comes with experience. Moreover, it must be noted that in our study, the ultrasound findings were not confirmed by histological sampling but with respect to the postoperative imaging (MRI in most cases). Knowing the limitations of postoperative MRI, we can argue that the accuracy of ultrasonography as judged on the postoperative MR images may not be entirely correct. An objective summary of the main pros and cons follows.

\section{Major Benefits}

1. Direct NUS eliminates the need for preoperative MRI. This saves time and money. It is also extremely useful in emergency cases where MRI may not be possible.

2. Direct NUS eliminates the registration inaccuracy inherent to image-to-patient registration algorithms, because the acquisition and display of the images is in the same reference frame.

3. The scans are very easy and convenient to obtain (not more than a few minutes each time an update is needed). Therefore, they can be repeated as many times as needed without significantly prolonging the overall duration of surgery.

4. Direct NUS does not interfere with the surgical workflow (unlike intraoperative MRI) and is not logistically challenging. This can be particularly convenient with awake surgery.

\section{Limitations}

1. Interpretation of the ultrasound images (in the ab- sence of a full head view) can be challenging for the lessexperienced user. By insonating a large volume to include surrounding brain and known anatomical landmarks (not just the tumor area), a large 3D volume can be reconstructed, and this considerably improves the orientation. Also, as mentioned, preoperative MR images can be used (in codisplay) in the early phase of the surgeon's learning curve.

2. Image quality may be suboptimal. Meticulous technique during insonation (mastered with experience over time) can minimize most of the causes of suboptimal image quality.

\section{Conclusions}

Direct navigated 3D ultrasound is a novel intraoperative imaging strategy for stand-alone navigation during tumor resective surgery. It provides quick, accurate, real-time updates and facilitates optimal resection with no major financial or logistical challenges. Experience with ultrasound image acquisition as well as interpretation is essential to extract the maximum benefit from this potentially useful adjunct.

\section{Acknowledgments}

We would like to thank Ms. Jino Joy, coordinator in the Tata Memorial Centre Neurosurgery Unit, for help in data collection and organization of the manuscript.

\section{References}

1. Aydin K, Kocabicak E, Altun A, Ozaydin I, Yarar E, Cokluk C: Use of topical landmarks for percutaneous projection of intracranial tumors for neurosurgical oncology. Turk Neurosurg 22:280-285, 2012

2. Barone DG, Lawrie TA, Hart MG: Image guided surgery for the resection of brain tumours. Cochrane Database Syst Rev 1:CD009685, 2014

3. Bozinov O, Burkhardt JK, Fischer CM, Kockro RA, Bernays RL, Bertalanffy H: Advantages and limitations of intraoperative 3D ultrasound in neurosurgery. Technical note. Acta Neurochir Suppl 109:191-196, 2011

4. Chacko AG, Kumar NK, Chacko G, Athyal R, Rajshekhar V: Intraoperative ultrasound in determining the extent of resection of parenchymal brain tumours - a comparative study with computed tomography and histopathology. Acta Neurochir (Wien) 145:743-748, 2003

5. Coburger J, König RW, Scheuerle A, Engelke J, Hlavac M, Thal DR, et al: Navigated high frequency ultrasound: description of technique and clinical comparison with conventional intracranial ultrasound. World Neurosurg 82:366375,2014

6. Coburger J, Scheuerle A, Thal DR, Engelke J, Hlavac M, Wirtz CR, et al: Linear array ultrasound in low-grade glioma surgery: histology-based assessment of accuracy in comparison to conventional intraoperative ultrasound and intraoperative MRI. Acta Neurochir (Wien) 157:195-206, 2015

7. Gronningsaeter A, Kleven A, Ommedal S, Aarseth TE, Lie T, Lindseth F, et al: SonoWand, an ultrasound-based neuronavigation system. Neurosurgery 47:1373-1380, 2000

8. Hata N, Dohi T, Iseki H, Takakura K: Development of a frameless and armless stereotactic neuronavigation system with ultrasonographic registration. Neurosurgery 41:608614, 1997

9. Keles GE, Lamborn KR, Berger MS: Coregistration accuracy and detection of brain shift using intraoperative sononavigation during resection of hemispheric tumors. Neurosurgery 53:556-564, 2003 
10. LeRoux PD, Winter TC, Berger MS, Mack LA, Wang K, Elliott JP: A comparison between preoperative magnetic resonance and intraoperative ultrasound tumor volumes and margins. J Clin Ultrasound 22:29-36, 1994

11. Lindner D, Trantakis C, Renner C, Arnold S, Schmitgen A, Schneider J, et al: Application of intraoperative 3D ultrasound during navigated tumor resection. Minim Invasive Neurosurg 49:197-202, 2006

12. Miller D, Benes L, Sure U: Stand-alone 3D-ultrasound navigation after failure of conventional image guidance for deepseated lesions. Neurosurg Rev 34:381-388, 2011

13. Miller D, Heinze S, Tirakotai W, Bozinov O, Sürücü O, Benes L, et al: Is the image guidance of ultrasonography beneficial for neurosurgical routine? Surg Neurol 67:579-588, 2007

14. Moiyadi A, Shetty P: Objective assessment of utility of intraoperative ultrasound in resection of central nervous system tumors: A cost-effective tool for intraoperative navigation in neurosurgery. J Neurosci Rural Pract 2:4-11, 2011

15. Moiyadi AV, Kannan S, Shetty P: Navigated intraoperative ultrasound for resection of gliomas: Predictive value, influence on resection and survival. Neurol India 63:727-735, 2015

16. Moiyadi AV, Shetty PM, Mahajan A, Udare A, Sridhar E: Usefulness of three-dimensional navigable intraoperative ultrasound in resection of brain tumors with a special emphasis on malignant gliomas. Acta Neurochir (Wien) 155:22172225,2013

17. Müns A, Meixensberger J, Arnold S, Schmitgen A, Arlt F, Chalopin C, et al: Integration of a 3D ultrasound probe into neuronavigation. Acta Neurochir (Wien) 153:1529-1533, 2011

18. Nabavi A, Black PM, Gering DT, Westin CF, Mehta V, Pergolizzi RS Jr, et al: Serial intraoperative magnetic resonance imaging of brain shift. Neurosurgery 48:787-798, 2001

19. Nimsky C, Ganslandt O, Cerny S, Hastreiter P, Greiner G, Fahlbusch R: Quantification of, visualization of, and compensation for brain shift using intraoperative magnetic resonance imaging. Neurosurgery 47:1070-1080, 2000

20. Peredo-Harvey I, Lilja A, Mathiesen T: Post-craniotomy neuronavigation based purely on intraoperative ultrasound imaging without preoperative neuronavigational planning. Neurosurg Rev 35:263-268, 2012

21. Pichlmeier U, Bink A, Schackert G, Stummer W: Resection and survival in glioblastoma multiforme: an RTOG recursive partitioning analysis of ALA study patients. Neuro Oncol 10:1025-1034, 2008

22. Prada F, Del Bene M, Mattei L, Lodigiani L, DeBeni S, Kolev V, et al: Preoperative magnetic resonance and intraoperative ultrasound fusion imaging for real-time neuronavigation in brain tumor surgery. Ultraschall Med 36:174-186, 2015

23. Renovanz M, Hickmann AK, Henkel C, Nadji-Ohl M, Hopf NJ: Navigated versus non-navigated intraoperative ultrasound: is there any impact on the extent of resection of highgrade gliomas? A retrospective clinical analysis. J Neurol Surg A Cent Eur Neurosurg 75:224-230, 2014

24. Roberts DW, Miga MI, Hartov A, Eisner S, Lemery JM, Kennedy FE, et al: Intraoperatively updated neuroimaging using brain modeling and sparse data. Neurosurgery 45:11991207, 1999

25. Rubin JM, Dohrmann GJ: Efficacy of intraoperative US for evaluating intracranial masses. Radiology 157:509-511, 1985

26. Rygh OM, Selbekk T, Torp SH, Lydersen S, Hernes TA, Unsgaard G: Comparison of navigated 3D ultrasound findings with histopathology in subsequent phases of glioblastoma resection. Acta Neurochir (Wien) 150:1033-1042, 2008
27. Sanai N, Berger MS: Glioma extent of resection and its impact on patient outcome. Neurosurgery 62:753-764, 264266, 2008

28. Selbekk T, Jakola AS, Solheim O, Johansen TF, Lindseth F, Reinertsen I, et al: Ultrasound imaging in neurosurgery: approaches to minimize surgically induced image artefacts for improved resection control. Acta Neurochir (Wien) 155:973-980, 2013

29. Sergeeva O, Uhlemann F, Schackert G, Hergeth C, Morgenstern U, Steinmeier R: Integration of intraoperative 3Dultrasound in a commercial navigation system. Zentralbl Neurochir 67:197-203, 2006

30. Shibata S, Kunieda T, Adachi H, Ueno Y, Kohara N, Sakai $\mathrm{N}$ : Preoperative localization of intracranial lesions with MRI using marking pills. Clin Neurol Neurosurg 113:854-858, 2011

31. Solheim O, Selbekk T, Jakola AS, Unsgård G: Ultrasoundguided operations in unselected high-grade gliomas-overall results, impact of image quality and patient selection. Acta Neurochir (Wien) 152:1873-1886, 2010

32. Unsgaard G, Gronningsaeter A, Ommedal S, Nagelhus Hernes TA: Brain operations guided by real-time two-dimensional ultrasound: new possibilities as a result of improved image quality. Neurosurgery 51:402-412, 2002

33. Unsgaard G, Rygh OM, Selbekk T, Müller TB, Kolstad F, Lindseth F, et al: Intra-operative 3D ultrasound in neurosurgery. Acta Neurochir (Wien) 148:235-253, 2006

34. Unsgaard G, Selbekk T, Brostrup Müller T, Ommedal S, Torp SH, Myhr G, et al: Ability of navigated 3D ultrasound to delineate gliomas and metastases-comparison of image interpretations with histopathology. Acta Neurochir (Wien) 147:1259-1269, 2005

35. van Velthoven V: Intraoperative ultrasound imaging: comparison of pathomorphological findings in US versus CT, MRI and intraoperative findings. Acta Neurochir Suppl 85:95-99, 2003

36. Wirtz CR, Albert FK, Schwaderer M, Heuer C, Staubert A, Tronnier VM, et al: The benefit of neuronavigation for neurosurgery analyzed by its impact on glioblastoma surgery. Neurol Res 22:354-360, 2000

37. Woydt M, Krone A, Becker G, Schmidt K, Roggendorf W, Roosen K: Correlation of intra-operative ultrasound with histopathologic findings after tumour resection in supratentorial gliomas. A method to improve gross total tumour resection. Acta Neurochir (Wien) 138:1391-1398, 1996

\section{Disclosures}

The authors report no conflict of interest concerning the materials or methods used in this study or the findings specified in this paper.

\section{Author Contributions}

Conception and design: Moiyadi. Acquisition of data: both authors. Analysis and interpretation of data: Moiyadi. Drafting the article: Moiyadi. Critically revising the article: Moiyadi. Reviewed submitted version of manuscript: both authors. Approved the final version of the manuscript on behalf of both authors: Moiyadi. Statistical analysis: Moiyadi. Administrative/ technical/material support: both authors. Study supervision: Moiyadi.

\section{Correspondence}

Aliasgar V. Moiyadi, Division of Neurosurgery, Tata Memorial Centre, Mumbai 400012, India. email: aliasgar.moiyadi@gmail. com. 